\title{
Functional and biomechanical evaluation of a completely recellularized stentless pulmonary bioprosthesis in sheep
}

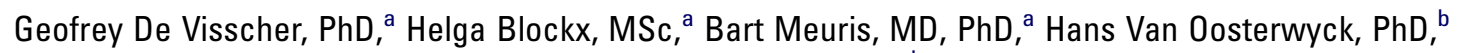
Erik Verbeken, $\mathrm{MD}, \mathrm{PhD},{ }^{c}$ Marie-Christine Herregods, $\mathrm{MD}, \mathrm{PhD},{ }^{\mathrm{d}}$ and Willem Flameng, $\mathrm{MD}, \mathrm{PhD}^{\mathrm{a}}$

From the Laboratory for Experimental Cardiac Surgery, Department of Cardiovascular Diseases, ${ }^{\text {a }}$ and Division of Biomechanics and Engineering Design, ${ }^{\mathrm{b}}$ Katholieke Universiteit Leuven, Leuven, Belgium; Department of Morphology and Molecular Pathology, University Hospital Leuven, Leuven, Belgium; and Department of Cardiology, University Hospital Leuven, ${ }^{\mathrm{d}}$ Leuven, Belgium.

The project was partially funded by Fonds voor Wetenschappelijk Onderzoek (G.0549.06).

Received for publication June 5, 2008; revisions received Aug 24, 2008; accepted for publication Sept 6, 2008.

Address for reprints: Geofrey De Visscher, $\mathrm{PhD}$, Laboratory for Experimental Cardiac Surgery, Dept. Cardiovascular Diseases, Katholieke Universiteit Leuven, Minderbroedersstraat 17,3000 Leuven, Belgium (E-mail: geofrey.devisscher@med.kuleuven.be).

J Thorac Cardiovasc Surg 2008;135:395-404 $0022-5223 / \$ 34.00$

Copyright $\odot 2008$ by The American Association for Thoracic Surgery

doi:10.1016/j.jtcvs.2007.09.016
Objective: In a previous study we showed that recellularization of a stentless bioprosthetic valve is stimulated 1 month after implantation in the pulmonary position, when its matrix (acellular photo-oxidized bovine pericardium) was preseeded by intraperitoneal implantation during a 3-day period.

Methods: The present study reports on the functional and biomechanical properties of such valves $(\mathrm{n}=19)$ in sheep up to 5 months after implantation. Similar valves $(\mathrm{n}=20)$ that were not intraperitoneally preseeded served as controls.

Results: Recellularization was partial in control valves and excessive in preseeded valves: $66 \%$ versus $223 \%$ of cellularity of native valves, respectively $(P<.05)$. The valves were endothelialized and contained interstitial cells depositing new matrix (collagens and elastin). However, phenotyping revealed an increased proportion of cells with contractile properties (30\%-40\% alpha smooth muscle actin ${ }^{+}$) in both groups. Intraperitoneally seeded valves had thicker and shorter leaflets that were associated with mildly increased peak gradients and regurgitation. Characterization of the matrix properties revealed a gradually degrading matrix ( $\pm 25 \%$ loss of collagen organization at 5 months) and a concomitant alteration of its biomechanical properties, that is, decreased strength, stiffness, and maximum force. However, overall valve function remained intact, and the biomechanical properties of the whole valves were superior to that of the native valves.

Conclusion: The ectopic in vivo seeding paradigm provides full recellularization. However, the volume fraction of the cellular phenotypes is not optimal, resulting in inadequate remodeling of the valves.

I $\mathrm{n}$ a recent overview, Rabkin and Schoen ${ }^{1}$ classified the paradigms used in cardiovascular tissue engineering. The classic in vitro paradigm describes the construction of a valve by combining cells and scaffold in vitro, followed by in vitro maturation of the construct in a bioreactor. However, according to Rabkin and Schoen, an alternative tissue-engineering strategy is to use scaffolds derived from natural materials that attract endogenous cells to repopulate and remodel the altered tissue. For this purpose, freeze-dried porcine valves, ${ }^{2}$ decellularized porcine valves, ${ }^{3}$ or isolated cell-free porcine small intestine submucosa (SIS) ${ }^{4}$ was used as a bioscaffold.

In a previous study, we used this concept of in vivo or endogenous cell seeding on a cross-linked but noncytotoxic, acellular scaffold to construct a heart valve. ${ }^{5}$ We used photo-oxidized bovine pericardium (POP) as a matrix, ${ }^{6}$ which was intraperitoneally (IP) seeded for 3 days, to construct a valve that was subsequently implanted in the pulmonary position. By orthotopic implantation of the valve, the heart itself functioned as a bioreactor to induce adequate differentiation of the endogenously seeded cells. This previous study mainly described the cellular aspects of the valves up to 1 month after their construction. It was shown that complete recellularization was obtained and associated with differentiation into a myofibroblast phenotype, concomitant neomatrix deposition, and adequate reendothelialization. 


\section{Abbreviations and Acronyms}

ASMA = alpha smooth muscle actin

DI $=$ deionized

IP = intraperitoneal

POP = photo-oxidized bovine pericardium

SIS $=$ small intestine submucosa

In the present study, we used the same model as that in our previous study, ${ }^{5}$ but the valves were followed up much longer, that is, to 5 months after implantation. The analysis focused more on functionality, durability, and biomechanical properties than on detailed cellular characterization. Nevertheless, the evolution of recellularization and neomatrix formation were studied during this maturation process. Therefore, data from the previous study are partially recapitulated in the actual report.

\section{Materials and Methods Animals}

Young purebred female "Lovenaar" sheep $(\mathrm{n}=40)$ were selected and allocated at random to 2 groups. Their average age was 516 \pm 169 days, and their average weight was $61.6 \pm 10.0 \mathrm{~kg}$. All animals were cared for by a veterinarian in accordance with the "Guide for the Care and Use of Laboratory Animals" (National Institutes of Health publication 85-23, revised 1985). The study was approved by the Ethics Committee of the Katholieke Universiteit Leuven.

There was no early or late mortality in both groups, but animals diagnosed with endocarditis were excluded from the study. This was the case in 1 animal in the 5-month IP preseeded group, and the animal was excluded from analysis.

\section{Surgery}

All sheep were premedicated with 10 to $20 \mathrm{mg} / \mathrm{kg}$ ketamine intramuscularly, followed by anesthetic induction with increasing concentrations of isoflurane in oxygen. After endotracheal intubation and institution of mechanical ventilation, anesthesia was maintained with isoflurane in $5 \mathrm{~L} / \mathrm{min} \mathrm{O}_{2}$ and $2 \mathrm{~L} / \mathrm{min}_{2} \mathrm{O}$.

Group 1 sheep $(n=19)$ had a pericardial patch of POP (Cardiofix, donated by Sulzer Carbomedics, Austin, Tex) implanted in the abdominal cavity. Three days later the sheep were again prepared for surgery as described above. The peritoneal cavity implant was aseptically retrieved without sacrificing the animals. The patch was folded and sutured into a stentless valve construct ${ }^{5,7}$ and kept in ice-cold saline until implantation in the pulmonary artery. During the valve construction, a left thoracotomy was performed at the third intercostal space. A cardiopulmonary bypass system was applied, and the in vivo seeded vascular construct was implanted as an interposition in the pulmonary artery. The native pulmonary valve was excised. The animals received analgesics (Dipidolor, Janssen Pharmaceutics, Beerse, Belgium) for the first 2 days and diuretics (Lasix, Aventis Pharma, Brussels, Belgium) when necessary. Genta-Kel $10 \%$ (Kela NV, Hoogstraten, Belgium) and Clexane (Aventis Pharma, Brussels, Belgium) were administered intramuscularly for 7 days after the operation.
During euthanasia, the animals were first premedicated with ketamine, and anesthesia was induced with isoflurane in oxygen. At this point a transthoracic echocardiography was performed (see below). After receiving heparin, the animals were administered an overdose of pentobarbital (Nembutal; Ovation Pharmaceuticals Inc, Deerfield, Ill) and $\mathrm{KCl}$. Subsequently the implants were removed aseptically and immediately fixed in $4 \%$ paraformaldehyde.

The following experimental protocol was followed:

1) Group 1: pulmonary implants of valves not previously IP seeded $(n=20)$ were explanted at 1 week $(n=6), 1$ month $(\mathrm{n}=6)$, and 5 months $(\mathrm{n}=8)$.

2) Group 2: pulmonary implants of valves previously IP seeded $(n=19)$ were explanted at 1 week $(n=6), 1$ month $(n=6)$, and 5 months $(n=7)$.

\section{Echocardiographic Analysis}

Before sacrifice or at 3 months, the animals were anesthetized and placed on the operation table in a right lateral recumbent position and attached to the anesthesia machine (Julian, Dräger, Germany). Sheep assigned to the 1-week and 1-month groups were assessed before sacrifice. Overall, data were obtained at 1 week, 1 month, 3 months, and 5 months. Echocardiography was performed by an experienced echocardiographer using a Vivid Five echo system (GE Medical Systems, Milwaukee, Wis) and a 2.5-MHz GE Ultrasound probe (GE Medical Systems). The peak gradient was assessed with continuous-wave Doppler, and the degree of regurgitation was assessed with color flow Doppler echocardiography.

\section{Biochemical and Biomechanical Valve Testing}

The valves explanted at 5 months were used for the following tests:

Calcium analysis. The tissue was lyophilized, weighed, and dissolved in a $20 \%$ hydrochloric acid solution $(10 \mathrm{mg}$ dried tissue $/ 1 \mathrm{~mL}$ $\mathrm{HCl})$ for 24 hours. After homogenization, samples were kept at $70^{\circ} \mathrm{C}$ overnight. Samples were then analyzed with a Calcium-kit (Chema Diagnostica, Monsano, Italy) and a spectrophotometer (Multiscan EX, Thermo Electron Corp, Woburn, Mass). Calcium levels were expressed as micrograms per milligram.

Degradation resistance. The following protease solution was used: $96 \mathrm{mg}$ of $\mathrm{CaCl}_{2} \cdot 2 \mathrm{H}_{2} \mathrm{O}$ and $97 \mathrm{mg}$ of protease (Type XIV from Streptomyces griseus) dissolved in $180 \mathrm{~mL}$ of buffer consisting of $0.01 \mathrm{~mol} / \mathrm{L}$ HEPES (Gibco, Gaithersburg, Md), $0.9 \%$ (m/v) NaCl (VWR, Heverlee, Belgium), and $0.1 \mathrm{~mol} / \mathrm{L}$ glycine (Fluka, Buchs, Switzerland). Lyophilized samples of known mass were incubated in the protease solution for 30 hours at $37^{\circ} \mathrm{C}$. After removal from the solution, samples were washed 3 times with deionized (DI) water (5 minutes) and lyophilized. Resistance toward protease degradation was given as the mass of remaining tissue expressed as a percentage of the predigestion mass.

Shrinkage temperature. A tensiometer (Force transducer type 372, Hugo Sachs, Germany) was used to measure the shrinkage temperature. Strips of tissue $(5 \mathrm{~mm})$ were sewed into rings and submerged in saline solution $(0.9 \% \mathrm{NaCl})$. The immersing solution was gradually heated from $25^{\circ} \mathrm{C}$ to $95^{\circ} \mathrm{C}$, and the temperature at the onset of shrinkage was considered as the shrinkage temperature.

Tensile mechanical properties. Tensile tests were performed on a custom-made uniaxial testing device comprising a $200 \mathrm{~N}$ load cell (Sensy, model 2712, Jumet, Belgium). Five-millimeter wide, full 
leaflet-length samples were clamped at an interclamp distance of 6 $\mathrm{mm}$. A monotonically increasing displacement was applied at a rate of $2 \mathrm{~mm} / \mathrm{min}$ until failure occurred. E-modulus, maximal force, and strength were calculated on the basis of force-displacement curves. Samples from control and IP seeded valves were tested immediately after sacrifice, and tests were performed at room temperature in ambient air.

Sirius red for collagen organization. The following Sirius red (SR) solution was prepared: $8 \mathrm{~g}$ picric acid (VWR) was dissolved in $200 \mathrm{~mL}$ DI water and mixed for 30 minutes; after filtration, $0.2 \mathrm{~g}$ old red (Aldrich Chemical Co, Milwaukee, Wis) was added, and the solution was filtered a second time. After immersion in DI water for 5 minutes, the frozen sections were submerged in the SR solution for 90 minutes. Sections were directly placed in $0.01 \mathrm{~N} \mathrm{HCl}$ and mounted. Sections were viewed using normal and polarized light to reveal the total collagen and organized collagen of the original POP, respectively. Collagen content was expressed as a percentage of total area, and organized collagen was expressed as a percentage of total collagen.

Sirius red/Fast green for collagen content. SR/Fast green solution was prepared as described above but with the addition of $0.2 \mathrm{~g}$ fast green FCF (Sigma, St Louis, Mo) before the final filtration. After immersion in DI water for 5 minutes, $0.2 \mathrm{~mL} \mathrm{SR/Fast} \mathrm{green}$ solution was added to the frozen sections for 30 minutes. Sections were washed with DI water and mounted or samples were scraped off the glass, added to $2 \mathrm{~mL}$ of $0.1 \mathrm{~N} \mathrm{NaOH}$ in methanol for $1 \mathrm{~min}$ ute, and centrifuged. The absorbance of the supernatant was determined at 605 and $540 \mathrm{~nm}$. The value corresponding to $29.1 \%$ of the optical density at $605 \mathrm{~nm}$ was calculated, representing the contribution of Fast green to the absorbance of SR at $540 \mathrm{~nm}$. The above value was subtracted from the absorbance at $540 \mathrm{~nm}$ to obtain the corrected absorbance. Next, the absorbance at $605 \mathrm{~nm}$ and the corrected absorbance at $540 \mathrm{~nm}$ were divided by their respective color equivalences ( 2.08 and 38.4, respectively) to obtain the net amount of collagen and noncollagenous protein in the section. Total protein is the sum of both values; the amount of collagen per mg of protein was calculated.

Assessment of residual amines. Frozen samples were lyophilized, weighed, and equilibrated in $2 \mathrm{~mL}$ of a $4 \% \mathrm{NaHCO}_{3}$ solution for 30 minutes. After reaction with $2 \mathrm{~mL} 0.5 \%$ TNBS (Sigma) at $40^{\circ} \mathrm{C}$, samples were rinsed with $0.9 \% \mathrm{NaCl}$ solution to remove unreacted TNBS. After 20 hours of hydrolysis in $2 \mathrm{~mL}$ of $25 \% \mathrm{HCl}$ at $100^{\circ} \mathrm{C}$, dilution by the addition of $8 \mathrm{~mL}$ of DI water, and extraction of unreacted TNBS with $2 \times 10 \mathrm{~mL}$ diethylether, the absorbance values of the hydrolysates were measured at $344 \mathrm{~nm}$. Residual amines were calculated using a molar absorption coefficient of $14.600 \mathrm{~mL} / \mathrm{mol}^{-1} / \mathrm{cm}^{-1}$ and expressed as micromoles of amines per milligram of dry tissue.

\section{Histologic Analysis and Immunohistochemistry}

After overnight fixation in $4 \%$ paraformaldehyde, the samples were incubated overnight in $20 \%$ sucrose in phosphate-buffered saline, imbedded in Neg-50 medium (Prosan, Merelbeke, Belgium), frozen, and stored at $-80^{\circ} \mathrm{C}$. Cryosectioning was performed on a Microm HM500 OM cryostat (Prosan). The $7-\mu \mathrm{m}$ sections were placed on poly-L-lysine-coated slides and stored at $-20^{\circ} \mathrm{C}$ until staining. Before immunohistochemistry was performed, the presence of cells was verified using standard hematoxylin and eosin staining and microscopic screening. The samples with cells present were further studied with immunofluorescent staining for vimentin (clone V9, Dako; Glostrup, Denmark), alpha smooth muscle actin (ASMA, clone 1A4, Dako), smooth muscle heavy chain myosin (clone SMMS-1, Dako), smoothelin (polyclonal; Santa Cruz Biotechnology, Santa Cruz, Calif), or endothelial nitric oxide synthase (clone 3, BD Pharmingen, San Diego, Calif). DAPI, a nuclear stain, was used for counterstaining. Microthrombi were scored on the hematoxylin and eosin-stained sections. The presence of elastin was assessed on von Giessen elastica-stained sections.

The total number of cells was counted on 1 complete leaflet section for each valve. DAPI-stained leaflets were digitally recorded, the micrographs were assembled in a mosaic image, and the nuclei were automatically counted using an Axioplan 2 imaging microscope and Axiovision 4.2 software package (Zeiss, Zaventem, Belgium). The same setup was used to perform morphometry and image analysis. For cell phenotyping, except for endothelial nitric oxide synthase, a total of 500 cells were assessed for each leaflet, and the results were expressed as a percentage. To avoid bias, several pictures for each leaflet were taken, quartered, and counted according to a randomization list.

\section{Statistical Analysis}

Generally, we used nonparametric statistics because of the limited number of observations in each group. Continuous data from the different groups were first compared with a Kruskal-Wallis test. If the obtained $P$ value was less than .05 , appropriate comparisons between the groups were performed by means of a Wilcoxon MannWhitney test. The echocardiographic data obtained at different time points were analyzed with a linear regression model. All of these tests were performed with the Statistical Package for the Social Sciences 14.0 for Windows (SPSS Inc, Chicago, Ill).

The score for calcification data was analyzed with a chi-square test. Because the regurgitation and microthrombus data were obtained at different time points, they were analyzed with stratified $2 \times 2$ tables and the Mantel-Haenszel inference statistic test. These analyses were performed with StatXact 4.0.1 software (Cytel Inc, Cambridge, Mass).

\section{Results \\ Hemodynamic Performance}

Figure 1 summarizes the hemodynamic data obtained from transthoracic echocardiography. As shown in the upper panel, the pressure gradient across the valve decreased progressively with time in both groups $(P=.008)$ but was higher in the IP seeded valves at all time points $(P=.040)$. These valves had also an increasing incidence of regurgitation with time $(P=.018)$. The degree of regurgitation, however, remained mild (ie, not $>2 / 4$ ).

\section{Macroscopic and Microscopic Valve Properties}

Macroscopic appearance. The IP valves were markedly remodeled, and the leaflets had a nest-like appearance similar to native valves, with the formation of pronounced commissures. The leaflets of the non-preseeded valves had a more abundant, billowing appearance (Figure 2). 
A

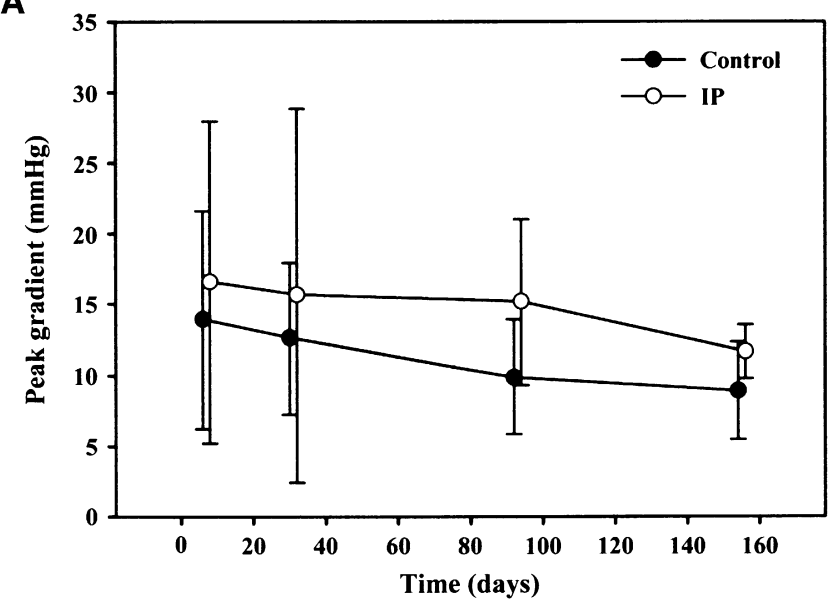

B

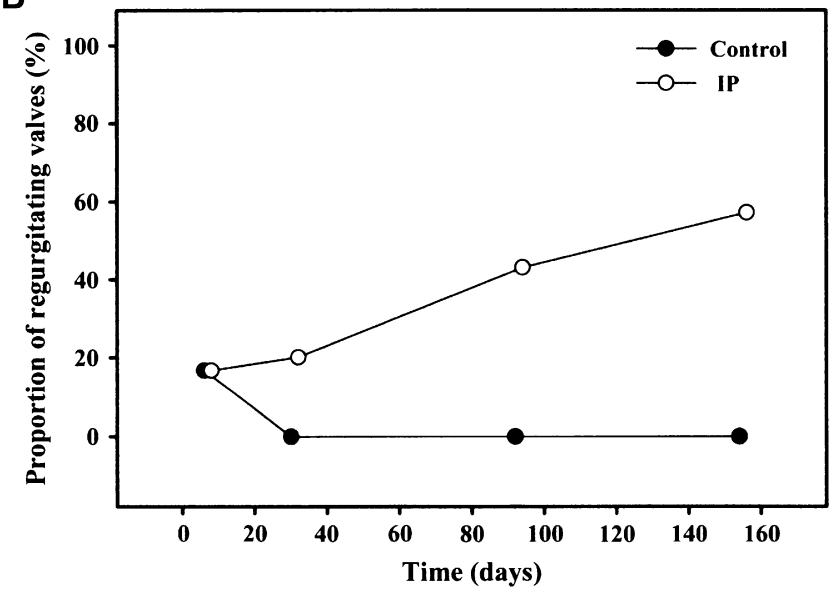

Figure 1. Hemodynamic assessment by transthoracic echocardiography. A, Peak pressure gradients across the pulmonary valve at 1 week, 1 month, 3 months, and 5 months after valve replacement. Linear regression: peak gradient $(P=.040)$ and time $(P=$ $.008)$ were shown to be significant factors. Mean and standard deviation (dots and bars). B, Incidence of pulmonary valve regurgitation (score $<{ }^{y} / 4$ ) at 1 week, 1 month, 3 months, and 5 months after valve replacement. Common odds ratio estimate: 11.27 (1.51; 84.22); homogeneity $P=.220$; odds ratio $=$ test; $P=.018$ (Mantel-Haenszel inference). IP, Intraperitoneal.

Leaflet morphology. The leaflets of control valves had a preserved length and a small increase in thickness (Figure 3, $A$ and $B$ ). Conversely, the IP seeded valves showed a gradual decrease in length, resulting in double the thickness of the non-preseeded valves through 5 months in the pulmonary position $(P=.021)$. A concomitant shortening of the IP valves was also observed resulting in a $17 \%(P=.004)$ and $32 \%$ $(P=.029)$ decrease in leaflet length at 1 and 5 months, respectively. The amount of newly deposited material was also measured in the sections and is represented as the
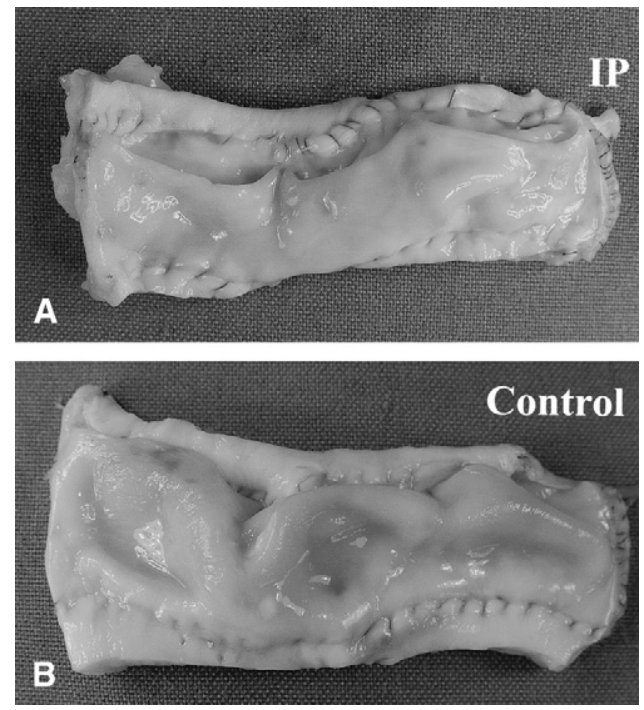

Figure 2. Macroscopic appearance of the valve explanted at 5 months. A, IP preseeded valve. Note the nest-like appearance of the leaflet. B, control (nonpreseeded valve). The leaflets are abundant and billowing. IP, Intraperitoneal.

percentage of the leaflet covered with new material (Kruskal-Wallis: $P<.001$ ) and the surface of new material (Kruskal-Wallis; $P=.002$ ). In controls, leaflet coverage was $28.9 \% \pm 29.8 \%$ and $11.7 \% \pm 8.3 \%$ at 1 week and 1 month, respectively, and increased significantly to $62.6 \% \pm 30.0 \%$ at 5 months $(P=.001 \mathrm{vs} 1$ month). The leaflet coverage of the IP valves, $90.0 \% \pm 16.8 \%, 65.7 \% \pm 28.8 \%$, and $89.8 \% \pm 26.5 \%$ at 1 week, 1 month and 5 months, respectively, did not differ significantly over time. However, it was significantly higher than that of the controls at each time point assessed $(P=.004, P=.002$, and $P=.009$, respectively). The tissue overgrowth data largely coincide with the previous data. The controls had a limited amount of new material, $3.57 \pm 4.92 \mathrm{~mm}^{2}, 1.17 \pm 0.80 \mathrm{~mm}^{2}$, and $1.74 \pm$ $1.39 \mathrm{~mm}^{2}$ at 1 week, 1 month, and 5 months, respectively. These values showed no significant difference. With exception of the $8.74 \pm 5.30 \mathrm{~mm}^{2}$ observed at 1 week, the $9.46 \pm$ $5.47 \mathrm{~mm}^{2}(P=.009)$ and $7.62 \pm 4.03 \mathrm{~mm}^{2}(P=.001)$ of new material in IP valves at 1 month and 5 months, respectively, were significantly higher than their time-related controls. Where present, the deposited new material stained positive for elastin in both groups, as did the native pulmonary valves (Figure 4, $A-C$ ).

Next, we studied the original matrix by assessing the amount and organization of the collagen by means of SRstained sections (Figure 5, Polarized Sirius Red). The scaffold's collagen density (Figure 3,C) remained stable in both groups at all time periods. However, although a significant yet small increase in the amount of organized collagen (Figure 3,D) in the control was noted at 1 week 
$(P=.041)$, a marked decrease in organization was observed in both the control and IP valves $(P=.001$ and .001 , respectively).

A limited presence of microthrombi could be observed in both groups at all time points, but the prevalence did not show a significant difference between the groups (MantelHaenszel inference: $P=.650$ ). The controls had 2/6, 1/6, and $3 / 8$, and IP valves had $3 / 6,2 / 6$, and $2 / 7$ microthrombipositive valves at 1 week, 1 month, and 5 months, respectively.

\section{Recellularization}

We found that the number of cells in the IP valve sections significantly exceeded that of the controls 4- to 5-fold at all time points $(P<.050)$ (Figures 3, $E$, and 5, Nuclei). However, we also measured the cells in sections of native pulmonary valves $(7482 \pm 1168$ cells/section) and plotted them in the same graph, thus showing that controls and IP valves were under- and overcellularized, respectively. Next we studied the presence of 4 markers: vimentin, ASMA, SMMS-1, and smoothelin, expressed by (myo)fibroblasts and smooth muscle cells (Table 1).

The fraction of cells that were positive for vimentin (a mesenchymal marker) and ASMA (a marker of myofibroblasts and smooth muscle cells) was equal in both the control and IP valves. For ASMA, the fractions of both groups significantly exceeded the proportion of these cells found in native pulmonary valves $(P<.001$ and $=.001$, respectively $)$. On the other hand, the fraction of smooth muscle cells, as assessed by SMMS-1 and smoothelin, was limited in control and IP valves only. All valves demonstrated re-endothelialization as shown by immunofluorescent staining for endothelial nitric oxide synthase (Figure $4, D-F$ ).

\section{Valve Characteristics at 5 Months of Implantation}

All the results of this section are summarized in Table 2. Calcification scores of electron-dense regions (Faxitron, Wheeling, Ill) showed that these were present in comparable proportions in both the control and IP valves. This finding was confirmed by absolute quantification of the calcium content. The values did not exceed those found in unimplanted matrix or the native pulmonary valve.

Protease degradation, a measure of the prosthesis' resistance to degradation, showed that the control and IP valves had a similar resistance as the unimplanted matrix. However, the native valves showed a significantly lower degradation resistance compared with the original matrix $(P<.001)$, control $(P=.011)$, and IP $(P=.002)$ valves. Collagen content was comparable among the original matrix, control, and IP valves. The free amines used to assess cross-linking efficiency showed low levels in unimplanted POP and equally increased levels in both control $(P=.001)$ and IP valves $(P=.001)$. However, they never attained the high level of free amines found in unfixed native pulmonary valves $(P=$ .016 and .002 , respectively).

The shrinkage temperature of the control $(P<.001)$ and IP valves $(P<.001)$ was significantly lower compared with that of the matrix material. The shrinkage temperature of native valves was significantly higher than that of the control valves $(P<.001)$, the IP valves $(P<.001)$, and even the POP $(P<.001)$. Mechanical tests showed a significant decrease in stiffness (E-modulus) in both control $(P=.013)$ and IP valves $(P<.001)$ compared with POP. The IP valves had a significantly lowered E-modulus compared with controls $(P=.008)$. Control valves had a significantly higher E-modulus than native valves $(P=.008)$, whereas IP valves were comparable to native valves. Because the E-modulus is calculated from the stress-strain curves combined with the sample's cross-section, one might argue that we do not have a homogeneous material and should therefore take into account only the original matrix. However, recalculating the E-modulus in this way $(5.30 \pm 2.69 \mathrm{MPa})$ did not change the findings. The maximal force attained before rupture (Fmax) showed a comparable yet significant decrease in Fmax of both control $(P=.004)$ and IP valves $(P=.008)$ compared with POP. However, they were both significantly higher than the Fmax from native valves $(P=.001$ and .002 , respectively). Strength showed significant decreases in control $(P=.001)$ and IP $(P<.001)$ valves compared with POP. Although no significant difference between both valve groups was detected, control $(P=.043)$ valves still had a significantly higher strength than the native valves, whereas IP valves were comparable to native valves. Here again, taking into account the original POP cross-section, the strength of the IP valves increased to $3.52 \pm 0.93 \mathrm{MPa}$, which became significantly higher than the values of the native valves $(P=.004)$.

\section{Discussion}

In the past, scaffolds derived from natural materials that attract endogenous cells, such as freeze-dried porcine valves, ${ }^{2}$ decellularized porcine valves, ${ }^{3}$ or isolated cell-free porcine SIS, ${ }^{4}$ were used in tissue engineering of heart valves. With respect to the freeze-dried porcine valves, the matrix properties were altered. Perforations allowing fibroblast infiltration and gentle abrasion of the surface to enhance endothelial cell adhesion showed positive results, ${ }^{2}$ but further in vivo evaluation is certainly necessary. Their protocol also killed the original porcine cells by a freeze-drying process, and within the 10-day culturing period the cell remnants disintegrated. ${ }^{2}$ Here again, in vivo studies are imperative because similar prostheses have shown detrimental inflammatory complications on implantation in patients. ${ }^{8}$ The groups of Elkins and colleagues $^{3}$ and Matheny and colleagues ${ }^{4}$ assessed recellularization of decellularized porcine valves and cell-free porcine SIS in the pulmonary position in sheep and swine, respectively. In both cases, the grafts became recellularized with 

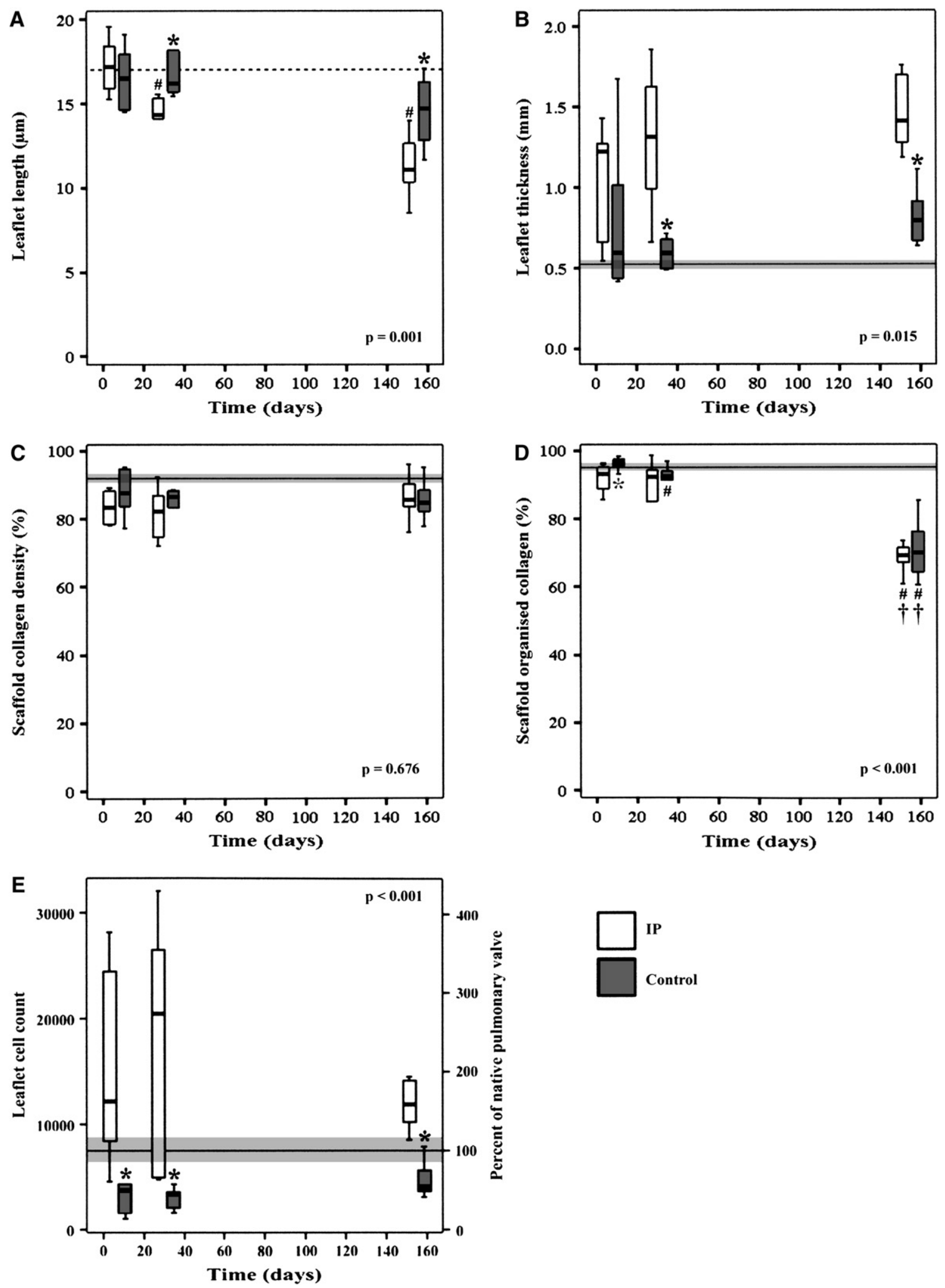

Time (days) 

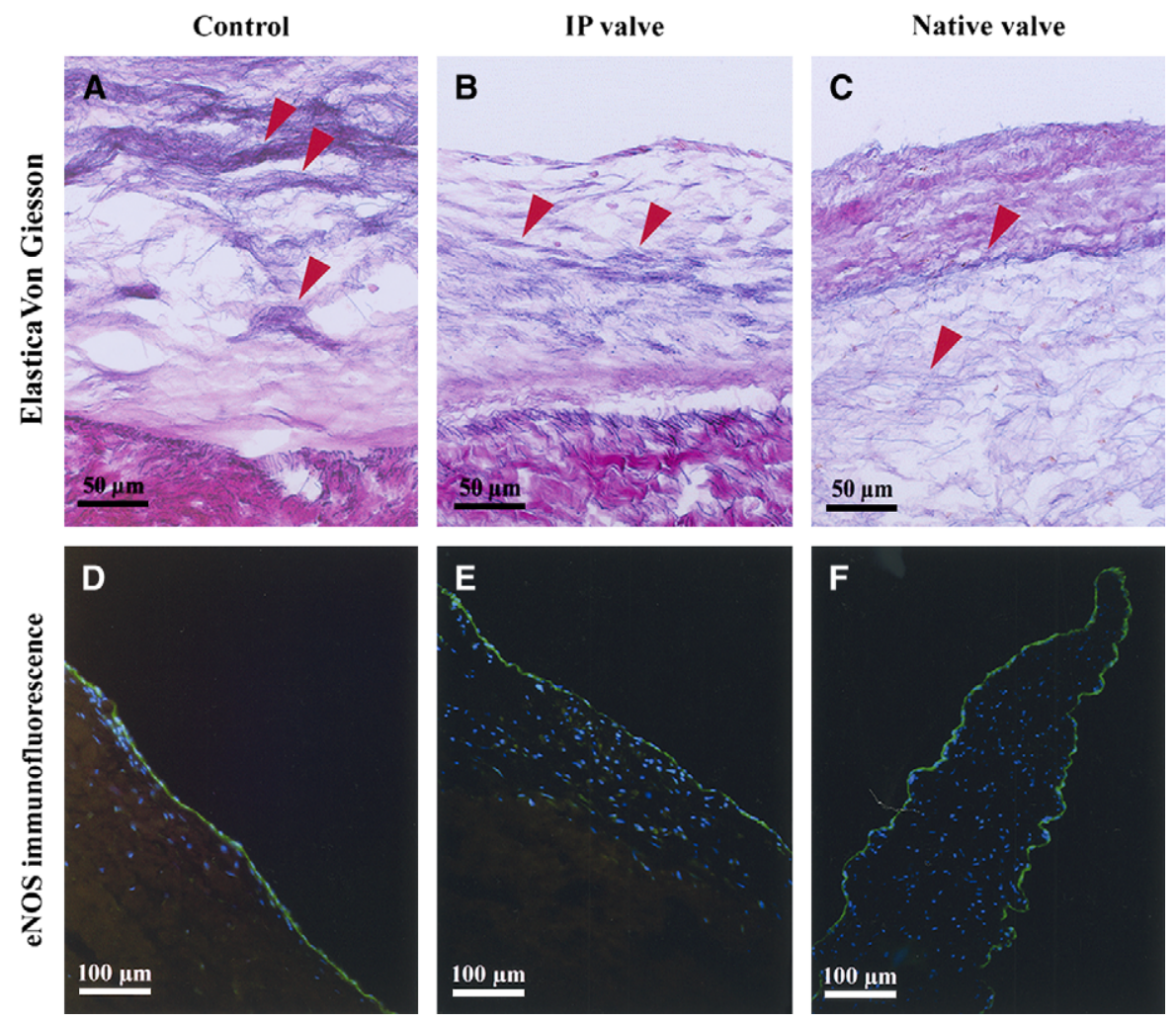

Figure 4. Representative images of Von Giessen stained (A-C) and endothelial nitric oxide synthase immunofluorescent (D-F) sections. The elastic fibers (A-C), present in all deposited material and the native valve, are visible as black stained fibers and examples are marked with arrows. The green fluorescence (D-F) represents the endothelial nitric oxide synthase positive cells, and the nuclei are counterstained with DAPI (b/ue dots). IP, Intraperitoneal; eNOS, endothelial nitric oxide synthase. largely the same cell populations. However, 1 major difference was that SIS, which was assessed after a shorter time in the pulmonary position, was resorbed and replaced by new matrix material. ${ }^{4}$ Also, handmade valves similar to the one used here made from SIS have been implanted in the pulmonary position in sheep. ${ }^{9}$ These valves showed immediate function, but only limited follow-up and functional analysis were performed.

The concept of IP seeding originates from a first attempt to grow an artificial artery graft out of granulation tissue, known as the "Sparks mandril" prosthesis. ${ }^{10}$ More recently, Campbell and coworkers ${ }^{11}$ showed the construction of a functional vascular graft generated from an IP deposited capsule around an implanted silastic tube. This tube (or foreign body) was left in the peritoneal cavity for several weeks until a complete encapsulation was obtained. After removal of the tube, they everted the capsule to internalize the exterior mesothelial layer and implanted the grafts in the abdominal aorta of both rats and rabbits. In this approach, the seeding is done by the graft recipient itself. The difference between our approach and that of Campbell and coworkers ${ }^{11}$ is that we did not leave the scaffold in the peritoneal cavity for the whole maturation process of encapsulation. We used only the initial IP "seeding phase," that is, at 3 days IP

Figure 3. Data are represented as box plots with the horizontal marker and box representing the median and interquartile range and the whiskers showing the distance from the end of the box to the largest and smallest observed values less than 1.5 box lengths from either end of the box. *Significant difference from the IP valves at the same time point. \#Significant difference from the 1-week group within the same seeding group. $\nmid$ Significant difference from the 1-month group within the same seeding group. The reported $P$ values are obtained from the Kruskal-Wallis test for multiple unpaired samples. A, Leaflet length measured on hematoxylin and eosin-stained cross-sections. The designed preimplant leaflet length (dashed line). B, Leaflet thickness measured on hematoxylin and eosinstained cross-sections. Thickness of unimplanted POP (line) and 95\% CI (gray area). C, Collagen density of the POP in the valves assessed by picrosirius red staining. Collagen density of unimplanted POP (line) and $95 \% \mathrm{Cl}$ (gray area). D, Organized collagen of the POP in the valves assessed by picrosirius red staining. The organized collagen of unimplanted POP (line) and the $95 \% \mathrm{CI}$ (gray area). E, Total cell count (left axis) of the valve leaflet and comparison with a native pulmonary heart valve (right axis). Total cell count of a native sheep pulmonary valve leaflet (line) and 95\% CI (gray area). IP, Intraperitoneal. 


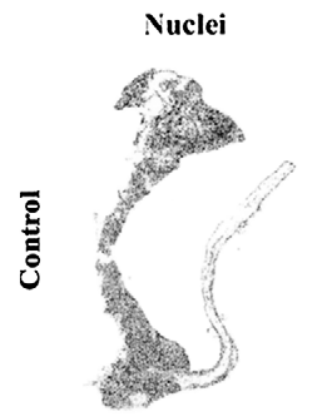

Polarised Sirius red
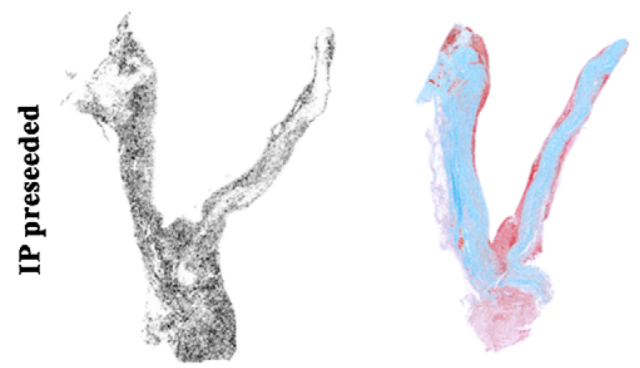

Figure 5. Representative images of nuclei density and picrosirius red stained sections of control and IP valves. The nuclei density maps were obtained by determining the $X$ and $Y$ coordinates of each individual nucleus on DAPI-stained sections and calculating a density map with the TOPCAT online software package (Mark Taylor, http://www.starlink.ac.uk/topcat/). The picrosirius red stained sections show the red stained collagen; in organized collagen bundles photographed with polarized light, the collagen is shown in blue pseudo-color. $I P$, intraperitoneal.

implantation, when there is a peak attraction of primitive cells. ${ }^{5,12}$ After 3 days we removed the scaffold from the peritoneal cavity and sutured it into a valve construct that was implanted in the pulmonary position for up to 5 months.

In our actual study, all valves remained functional for at least 5 months. However, the valves that were IP preseeded had a consistently higher peak gradient and an increased tendency for regurgitation. The likely reason for both these features was the excess cellularization after IP preseeding compared with controls, which was observed immediately after implantation. Both groups also showed bidirectional variation in recellularization (data not shown). In addition to recellularization, we also found a significant amount of new matrix material deposited on the photo-oxidized scaffold of IP valves. This new material approximately doubled the leaflet cross-sectional surface and therefore inherently its mass. This increased resistance explains the observed higher peak gradients. The increase in new material could not be attributed to thrombosis because both groups were minimally thrombogenic. The gradual decrease of peak gradients over time is expected in stentless valves. ${ }^{13}$ With respect to the increased regurgitation, we observed extensive shortening of the leaflets in the IP valves, resulting in inadequate coaptation. The reason behind the shrinkage became apparent when the sections were stained for the presence of myofibroblasts and smooth muscle cells. These cells with contractile properties normally represent only a minority of cells in a native leaflet, and almost all other cells are fibroblasts. In native pulmonary valves, only $2 \%$ to $5 \%$ of these cells are myofibroblasts and almost none are smooth muscle cells,${ }^{14}$ although increased amounts of these cells have been observed in pathologic conditions ${ }^{15,16}$ and in vitro cultured interstitial valve cells. ${ }^{17,18}$ In our valve constructs, be it preseeded or spontaneously recellularized, an excess of cells stained for ASMA. In the preseeded valves, we found the same for smoothelin. Although both valve constructs, controls and preseeded, had a significantly increased proportion of ASMA or smoothelin-positive cells, leaflet shortening was only observed in preseeded valves. This can be explained by the difference in degree of recellularization. In the valves left to recellularize spontaneously, significantly less cells, leaflet coverage, and new matrix deposition were found. Conversely, preseeded valves were excessively recellularized, resulting in a severe overshoot of the absolute number of cells with contractile properties. Although this may explain the $15 \%$ to $20 \%$ shortening of the leaflets of the preseeded valve construct, inducing malcoaptation, the leaflets still moved freely and functioned well. Obviously this process of recellularization is completely different from the process of excessive pannus overgrowth, as can be seen in some glutaraldehydefixed bioprostheses, called "open regurgitation." This is found in pediatric patients and is a result of exaggerated pannus formation overgrowing the leaflets and adhering them to the wall. ${ }^{19}$ It should also be considered that the valves were constructed with almost no coaptation area, and therefore

TABLE 1. Immunofluorescent staining

\begin{tabular}{|c|c|c|c|c|}
\hline & Control valves & IP valves & Native valves & $P$ value \\
\hline Vimentin (\%) & $46.9 \pm 14.9$ & $38.5 \pm 16.9$ & ND & .336 \\
\hline ASMA (\%) & $39.0 \pm 16.2^{*}$ & $30.5 \pm 19.5^{*}$ & $1.4 \pm 0.9$ & .001 \\
\hline SMMS-1 & $0.00 \pm 0.00$ & $0.00 \pm 0.00$ & $0.00 \pm 0.00$ & 1.000 \\
\hline Smoothelin & $1.42 \pm 1.53^{*}$ & $3.62 \pm 2.43^{*}$ & $0.00 \pm 0.00$ & .005 \\
\hline
\end{tabular}

$I P$, Intraperitoneal; ASMA, alpha smooth muscle actin; $N D$, not determined. Data are presented as mean \pm standard deviation. ${ }^{*}$ Significant differences from native valve. 
TABLE 2. Matrix properties

\begin{tabular}{lccccc}
\hline & POP & Control valves & IP valves & Native valves & P value \\
\hline $\begin{array}{l}\text { I. Calcification } \\
\text { X-ray score }\end{array}$ & ND & $5 / 8$ & & & \\
$\quad$ Ca content $(\mu \mathrm{g} / \mathrm{mg})$ & $0.62 \pm 1.10$ & $3.37 \pm 2.75$ & $3.03 \pm 3.70$ & $3.86 \pm 5.00$ & $.085\left(\chi^{2}\right)$ \\
II. Biochemical properties & & & & \\
$\quad$ Protease degradation (\%) & $65.2 \pm 2.9^{*}$ & $58.5 \pm 6.8^{*}$ & $63.1 \pm 5.0^{*}$ & $40.8 \pm 14.7$ & $<.001(\mathrm{KW})$ \\
$\quad$ Collagen content $(\mu \mathrm{g} / \mathrm{mg})$ & $51.1 \pm 17.7$ & $40.5 \pm 19.6$ & $38.3 \pm 19.2$ & $\mathrm{ND}$ & $.221(\mathrm{KW})$ \\
$\quad$ Free amines $(\mu \mathrm{mol} / \mathrm{mg})$ & $20.9 \pm 1.4^{*}$ & $90.9 \pm 34.3^{*} \dagger$ & $76.8 \pm 13.4^{*} \dagger$ & $200.0 \pm 132.5 \dagger$ & $<.001(\mathrm{KW})$ \\
III. Biophysical properties & & & & \\
$\quad$ Shrinkage temperature $\left({ }^{\circ} \mathrm{C}\right)$ & $59.4 \pm 0.7^{*}$ & $56.1 \pm 0.9^{*} \dagger$ & $56.1 \pm 1.2^{*} \dagger$ & $63.3 \pm 0.8 \dagger$ & $<.001(\mathrm{KW})$ \\
E (MPa) & $19.43 \pm 6.18^{*}$ & $11.56 \pm 6.99^{*} \dagger$ & $3.67 \pm 2.33 \dagger \dagger$ & $3.37 \pm 1.91 \dagger$ & $<.001(\mathrm{KW})$ \\
F max (N) & $25.8 \pm 9.1^{*}$ & $15.4 \pm 6.6^{*} \dagger$ & $14.6 \pm 6.0^{*} \dagger$ & $2.4 \pm 1.0 \dagger$ & $<.001(\mathrm{KW})$ \\
Strength (MPa) & $8.28 \pm 2.60^{*}$ & $4.08 \pm 2.10^{*} \dagger$ & $2.33 \pm 1.07 \dagger$ & $1.56 \pm 0.65 \dagger$ & $<.001(\mathrm{KW})$ \\
\hline
\end{tabular}

POP, Photo-oxidized bovine pericardium; $I P$, intraperitoneal; $N D$, not determined. Data are presented as mean \pm standard deviation. ${ }^{*}$ Significant difference from native valve. $\dagger$ Significant difference from unimplanted matrix material (Cardiofix, donated by Sulzer Carbomedics, Austin, Tex). $\ddagger$ Significant difference from spontaneous seeded valve. For the tensile strength-related variable, the correction was made with the original matrix only.

the slightest shrinkage of the leaflets will induce malcoaptation. Another crucial cell type in a normal native valve is the endothelial cell. As shown previously, IP seeding leads to reendothelialization, most probably by attraction of endothelial progenitors from the circulation. ${ }^{5}$ At 5 months this observation was confirmed, as was endothelialization of the pannus growing on the control valves.

Calcification, a major problem with commercially available valves, ${ }^{20,21}$ is determined by several factors, as previously reported; ${ }^{22}$ this includes the material used to make the valve prosthesis. The fact that photo-oxidized pericardium is resilient to calcification ${ }^{23,24}$ was confirmed, and no difference in calcification among the unimplanted matrix, spontaneously seeded, and IP valves was observed. Furthermore, the IP valves had a calcium content comparable to that of native sheep pulmonary valves.

In heart valve tissue engineering, a slowly degradable scaffold is preferred because the construct must withstand the stress implied by the circulation immediately after implantation and in the initial phase of recellularization. The photo-oxidized scaffold slowly degrades, as shown by the severe decrease in collagen organization of the matrix at 5 months. However, the collagen concentration and density remain unaltered. This clearly shows that when new matrix is deposited, it contains comparable amounts of collagen as the matrix. The photo-oxidized pericardium is more prone to slow degradation than glutaraldehyde-fixed pericardium. This is shown by resistance to degradation, which is lower for photo-oxidized than for glutaraldehyde-fixed pericardium (data not shown). This degradation occurs in the controls and the preseeded valves, and clearly affects the biophysical properties of the valves. As discussed above, changes in shrinkage temperature were observed, but more pertinent decreases in stiffness, maximal force, and strength were found. The decrease in maximal force was equal in both spontaneous and IP valves. Although only significant in the stiffness, both stiffness (E-modulus) and strength were decreased even further in IP preseeded valves. Because the IP valves had a larger cross-section because of the new material, we also adjusted the calculation by only taking into account the thickness of the original matrix. This resulted in increases in the obtained E-modulus and strength values, but even then the stiffness of the IP valves remained significantly lower than that of controls. Nevertheless, the biophysical properties of the valve constructs were still superior to those of the native pulmonary valves at 5 months after implantation. Two additional variables, the shrinkage temperature and amount of free amines, could contribute to evidence of degradation, but this could also be contributed to the deposition of new extracellular matrix material. Shrinkage temperature, a hallmark for crosslinking, showed a small but significant decrease after implantation with or without preseeding. Whether this decrease is attributed to degradation or a lower shrinkage temperature of a possibly less structured new matrix could not be accurately measured by our techniques. However, a biphasic shrinkage profile, measurable in composite materials or genipin-fixed pericardium subcutaneously implanted in rats, ${ }^{25}$ could not be observed. The large increase in free amines observed in controls and IP seeded valves can similarly be attributed to degradation and the deposition of new material containing increased amounts of free amines.

A limitation of the technology used to determine these biophysical properties is that we did not account for the anisotropy. This was impossible because we did not have enough material to perform additional tensile tests in the transverse direction. However, none of the obtained measurements were below that of a native pulmonary valve assessed in the same direction. In this respect it would also have been interesting to obtain the mechanical properties of the new matrix deposited by the cells. 


\section{Conclusions}

Our findings confirm that IP seeding leads to recellularization of heart valve constructs. However, the recellularization was excessive, and the increased amount of cells with contractile properties resulted in shortening of the leaflet and mild, yet progressive, regurgitation of these valves. From these experiments it became obvious that the problem with this seeding protocol is not the excessive recellularization, although it influenced the pressure gradients across the valve, but the increased amount of cells with contractile properties. At present it is not clear whether the retraction of the leaflets is ongoing. Theoretically, modified valve design with more redundancy of the leaflets could improve coaptation after valve maturation. However, we think that the search for an adapted seeding protocol optimizing valve cell type distribution is mandatory.

The authors thank laboratory assistants Ruth Plusquin, Veerle Leunens, Monique Vercalsteren, and Kristof Reyniers.

\section{References}

1. Rabkin E, Schoen FJ. Cardiovascular tissue engineering. Cardiovasc Pathol. 2002;11:305-17.

2. Curtil A, Pegg DE, Wilson A. Repopulation of freeze-dried porcine valves with human fibroblasts and endothelial cells. J Heart Valve Dis. 1997;6:296-306.

3. Elkins RC, Goldstein S, Hewitt CW, Walsh SP, Dawson PE, Ollerenshaw JD, et al. Recellularization of heart valve grafts by a process of adaptive remodeling. Semin Thorac Cardiovasc Surg. 2001;13(4 Suppl 1):87-92.

4. Matheny RG, Hutchison ML, Dryden PE, Hiles MD, Shaar CJ. Porcine small intestine submucosa as a pulmonary valve leaflet substitute. J Heart Valve Dis. 2000;9:769-74.

5. De Visscher G, Vranken I, Lebacq A, Van Kerrebroeck C, Ganame J, Verbeken E, et al. In vivo cellularisation of a cross-linked matrix by intraperitoneal implantation: a new tool in heart valve tissue engineering. Eur Heart J. 2007;28:1389-96.

6. Moore MA. PhotoFix: unraveling the mystery. J Long Term Eff Med Implants. 2001;11:185-97.

7. White JK, Agnihotri AK, Latremouille C, Messas E, Carpentier A, Torchiana DF. A method of using the pulmonary trunk to form a trileaflet valve. J Thorac Cardiovasc Surg. 2005;129:677-9.

8. Simon P, Kasimir MT, Seebacher G, Weigel G, Ullrich R, SalzerMuhar U, et al. Early failure of the tissue engineered porcine heart valve
SYNERGRAFT in pediatric patients. Eur J Cardiothorac Surg. 2003; 23:1002-6.

9. White JK, Agnihotri AK, Titus JS, Torchiana DF. A stentless trileaflet valve from a sheet of decellularized porcine small intestinal submucosa. Ann Thorac Surg. 2005;80:704-7.

10. Simmons P, Fogarty TJ, Pennell JP. Nineteen months clinical experience with the Sparks mandril graft. J Am Assoc Nephrol Nurses Tech. 1975;2: 120-3.

11. Campbell JH, Efendy JL, Campbell GR. Novel vascular graft grown within recipient's own peritoneal cavity. Circ Res. 1999;85:1173-8.

12. Campbell JH, Efendy JL, Han C, Girjes AA, Campbell GR. Haemopoietic origin of myofibroblasts formed in the peritoneal cavity in response to a foreign body. J Vasc Res. 2000;37:364-71.

13. Park SZ, Reardon MJ. Current status of stentless aortic xenografts. Curr Opin Cardiol. 2000;15:74-81.

14. Mendelson K, Schoen FJ. Heart valve tissue engineering: concepts, approaches, progress, and challenges. Ann Biomed Eng. 2006;34:1799-819.

15. Rabkin E, Aikawa M, Stone JR, Fukumoto Y, Libby P, Schoen FJ. Activated interstitial myofibroblasts express catabolic enzymes and mediate matrix remodeling in myxomatous heart valves. Circulation. 2001; 104:2525-32.

16. Schurch W, Seemayer TA, Gabbiani G. The myofibroblast: a quarter century after its discovery. Am J Surg Pathol. 1998;22:141-7.

17. Taylor PM, Batten P, Brand NJ, Thomas PS, Yacoub MH. The cardiac valve interstitial cell. Int J Biochem Cell Biol. 2003;35:113-8.

18. Yperman J, De Visscher G, Holvoet P, Flameng W. Molecular and functional characterization of ovine cardiac valve-derived interstitial cells in primary isolates and cultures. Tissue Eng. 2004;10:1368-75.

19. Murphy SK, Rogler WC, Fleming WH, McManus BM. Retraction of bioprosthetic heart valve cusps: a cause of wide-open regurgitation in right-sided heart valves. Hum Pathol. 1988;19:140-7.

20. Walther T, Falk V, Autschbach R, Diegeler A, Rauch T, Weigl C, et al. Comparison of different anticalcification treatments for stentless bioprostheses. Ann Thorac Surg. 1998;66(6 Suppl):S249-54.

21. Fiddler GI, Gerlis LM, Walker DR, Scott O, Williams GJ. Calcification of glutaraldehyde-preserved porcine and bovine xenograft valves in young children. Ann Thorac Surg. 1983;35:257-61.

22. Flameng W, Meuris B, Yperman J, De Visscher G, Herijgers P, Verbeken E. Factors influencing calcification of cardiac bioprostheses in adolescent sheep. J Thorac Cardiovasc Surg. 2006;132:89-98.

23. Adams AK, Moore MA. Calcification resistance biostability and low immunogenic potential of porcine heart valves stabilized by dye-mediated photooxidation. Trans Soc Biomater. 1999;22:312. Abstract.

24. Moore MA, McIlroy BK, Bianco RW, Phillips RE, Jr. Improved calcification performance of heart valve material prepared by dye-mediated photooxidation. In: Gabbay S, Wheatley DJ, eds. Advances in anti-calcific and anti-degenerative treatment of heart valve bioprostheses. 1st ed. Austin, TX: Silent Partners; 1997. p. 125-36.

25. Chang Y, Lee MH, Liang HC, Hsu CK, Sung HW. Acellular bovine pericardia with distinct porous structures fixed with genipin as an extracellular matrix. Tissue Eng. 2004;10:881-92. 